\title{
Impregnação de catalisadores em suporte de espuma cerâmica de alumina
}

\section{(Impregnation of catalysts on support of alumina ceramic foam)}

\author{
G. S. Falk', A. L. Rodriguez', W. Acchar', E. L. Foletto \\ ${ }^{1}$ Programa de Pós Graduação em Tecnologia Ambiental,Universidade de Santa Cruz do Sul, Santa Cruz do Sul, \\ RS, Brasil 96.815-900 \\ ${ }^{2}$ Departamento de Física, Universidade Federal do Rio Grande do Norte, Natal, RN, Brasil 59.072-970 \\ ${ }^{3}$ Departamento de Engenharia Química, Universidade Federal de Santa Maria, \\ Santa Maria, RS, Brasil 97.105-900 \\ efoletto@gmail.com
}

\begin{abstract}
Resumo
Este trabalho teve por objetivo avaliar a impregnação de diferentes catalisadores na superfície de um material cerâmico de alumina. Para a confecção do material cerâmico, foi utilizada a rota de processamento de espumas cerâmicas pelo método da réplica, no qual uma esponja de poliuretano com porosidade de 60 ppi foi mergulhada em uma suspensão de alumina a fim de produzir uma cerâmica com a mesma morfologia que o material precursor. Inicialmente foram obtidas espumas cerâmicas de alumina com resistência mecânica média de $0,7 \mathrm{MPa}$ e porosidade aparente de $80 \%$. Três diferentes catalisadores $\left(\mathrm{ZnAl}_{2} \mathrm{O}_{4}, \mathrm{ZnFe}_{2} \mathrm{O}_{4}\right.$ e $\left.\mathrm{Zn}_{2} \mathrm{SnO}_{4}\right)$ foram impregnados sobre esse suporte por meio do método dip coating.Os resultados mostraram que os catalisadores foram imobilizados sobre o suporte em baixas concentrações.
\end{abstract}

Palavras-chave: espuma cerâmica, alumina, método da réplica, impregnação, catalisadores.

\begin{abstract}
This study aimed to evaluate the immobilization of different catalysts on the surface of ceramic alumina foam. The ceramic material was produced by the replica method, where a polyurethane sponge with a porosity of 60 ppi was poured into a slurry containing alumina to produce a ceramic support with the same morphology of the precursor material. Alumina ceramic foams with an average strength of $0.7 \mathrm{MPa}$ and apparent porosity of $80 \%$ were obtained. Three different catalysts $\left(\mathrm{ZnAl}_{2} \mathrm{O}_{4}, \mathrm{ZnFe}_{2} \mathrm{O}_{4}\right.$ and $\left.\mathrm{Zn}_{2} \mathrm{SnO} \mathrm{O}_{4}\right)$ were impregnated on this support by the dip coating method. The results showed that the catalysts were immobilized on the substrate at low concentrations.
\end{abstract}

Keywords: ceramic foam, alumina, replica method, impregnation catalysts.

\section{INTRODUÇÃO}

A poluição ambiental no contexto atual chamou a atenção para a necessidade do desenvolvimento de novos processos, novas técnicas, novas tecnologias que permitam ao homem controlar e remediar de maneira mais eficaz os danos que o mesmo vem causando ao ecossistema. Nesse cenário, o estudo, o desenvolvimento e a aplicação de materiais para aplicações ambientais, tornam-se fundamentais não só para o monitoramento como também para a remediação de ambientes contaminados. Nesse contexto, materiais cerâmicos porosos vêm sendo estudados como meio suporte de catalisadores para tratamento de água e purificação de ar [1-3], além de uso em diversas reações químicas [4-7]. Esses materiais caracterizam-se por apresentar propriedades especificas, como elevada área superficial, baixa densidade, baixa condutividade térmica, permeabilidade controlada aliada a suas propriedades mecânicas [8], que os tornam interessante para a utilização como meio filtrante, adsorvente e suporte catalítico para tratamentos ambientais e diversas reações químicas.

As espumas cerâmicas são estruturas porosas com alta resistência mecânica e podem ser preparadas a partir de diferentes técnicas como réplica, gelcasting de espumas cerâmicas, queima de partículas orgânicas, dentre outras [9]. Neste trabalho foi usado o método de réplica para a obtenção da espuma cerâmica de alumina. Esse método consiste na imersão de um molde, nesse caso, uma esponja de poliuretano, em uma suspensão cerâmica, a qual é posteriormente seca e calcinada para remoção do material polimérico, mantendo o material cerâmico formado com uma estrutura similar a da esponja precursora [9].

Óxidos espinélios ternários tais como aluminato de zinco $\left(\mathrm{ZnAl}_{2} \mathrm{O}_{4}\right)$, ferrita de zinco $\left(\mathrm{ZnFe}_{2} \mathrm{O}_{4}\right)$ e estanato de zinco $\left(\mathrm{Zn}_{2} \mathrm{SnO}_{4}\right)$ tem sido largamente usados como catalisadores em diversas reações químicas [10-12], além da sua aplicação como fotocatalisadores na área ambiental para o tratamento de águas residuais[13-15]. Geralmente, esses 
são usados como catalisador na forma de pó em suspensão, o que traz como desvantagem a necessidade de separação e recuperação, o qual pode ser feita, por exemplo, por filtração. Uma alternativa para resolver esse problema é o uso de catalisador na forma imobilizada, como é o caso da imobilização sobre espumas cerâmicas. Diversos métodos para recobrimento da superfície do suporte cerâmico podem ser empregados tais como dip coating, spin coating e spraying [16]. Neste trabalho, os catalisadores foram imobilizados sobre a espuma cerâmica através do uso da técnica dip coating, que consiste na imersão do suporte cerâmico na solução precursora contendo o material a impregnar, por tempo e velocidades controladas. Esse método é simples e forma um recobrimento fino e homogêneo sobre a superfície da espuma cerâmica. Neste contexto, o principal objetivo do estudo foi preparar um suporte de espuma cerâmica de alumina para avaliar a posterior imobilização de diferentes materiais catalisadores tais como $\mathrm{ZnAl}_{2} \mathrm{O}_{4}, \mathrm{ZnFe}_{2} \mathrm{O}_{4}$ e $\mathrm{Zn}_{2} \mathrm{SnO}_{4}$. O suporte cerâmico foi obtido pela rota de processamento de espumas cerâmicas, através do método da réplica. A impregnação dos catalisadores foi realizada através do método de dip coating e foi avaliada por meio de análises de difração de raios $\mathrm{X}$, microscopia eletrônica de varredura e espectroscopia de energia dispersiva.

\section{MATERIAIS E MÉTODOS}

\section{Preparação da espuma cerâmica}

Esponjas de poliuretano, com concentração de poros linear de 60 ppi, da linha PentaCell, fabricada pela KoeppSchaumGmbH (Alemanha), com densidade $28 \mathrm{~kg} . \mathrm{m}^{-3}$, resistência a tração $100 \mathrm{kPa}$ e um alongamento até a ruptura de $150 \%$, foram escolhidas para este estudo. O material cerâmico utilizado foi a alumina calcinada $\left(\mathrm{Al}_{2} \mathrm{O}_{3}\right)$, fornecido pela Alcoa, Brasil. Para distribuição das partículas foram utilizadas peneiras 200 mesh. Na preparação da suspensão de alumina foi utilizado como solvente água destilada, como meio ligante solução de álcool polivinilíco P.S. (PVA) $\left(\mathrm{C}_{2} \mathrm{H}_{4} \mathrm{O}\right)$ n (Cromoline, Química Fina, Brasil), como agente dispersante solução de silicato de sódio (dosagem $\left(\mathrm{Na}_{2} \mathrm{O}\right)$ 20-30\% e dosagem $\left(\mathrm{SiO}_{2}\right)$ 20-30\%) (Vetec, Brasil).

$\mathrm{A}$ adição de plastificantes e ligantes na suspensão inicial foi para proporcionar revestimentos cerâmicos suficientemente fortes para evitar e ou diminuir a fissuração das espumas durante a pirólise do molde polimérico, que é um dos principais problemas do método réplica [17].

A preparação da suspensão cerâmica seguiu o seguinte procedimento descrito a seguir. $45 \mathrm{~mL}$ de água destilada, $8 \mathrm{~mL}$ solução de PVA e $2 \mathrm{~mL}$ de solução de silicato de sódio foram adicionados em $148 \mathrm{~g}$ de pó de alumina. Para a total mistura da suspensão cerâmica foi utilizado um misturador de hélices, com rotação de $\sim 500 \mathrm{rpm}$, durante $1 \mathrm{~h}$. No método, os moldes poliméricos foram imersos na suspensão cerâmica até total preenchimento dos poros. Em seguida, os moldes foram passados por rolos préajustados com espaçamento de $20 \%$ da altura do molde de poliuretano inicial para a retirada do excesso da suspensão cerâmica [18]. As espumas revestidas com a suspensão cerâmica passaram por uma secagem ambiente por $24 \mathrm{~h}$, sendo posteriormente aquecidas a $400{ }^{\circ} \mathrm{C}$ por $1 \mathrm{~h}$, a uma taxa de aquecimento de $1{ }^{\circ} \mathrm{C} \cdot \mathrm{min}^{-1}$. Subseqüentemente, as amostras foram aquecidas a $900{ }^{\circ} \mathrm{C}$ por $1 \mathrm{~h}$ a uma taxa de aquecimento de $2{ }^{\circ} \mathrm{C} \cdot \mathrm{min}^{-1}$ a fim de produzir pré-formas reticuladas com resistência ao manuseio. Após a secagem, as pré-formas foram sinterizadas a $1500{ }^{\circ} \mathrm{C}$ durante $2 \mathrm{~h}$ a uma taxa de aquecimento de $5^{\circ} \mathrm{C} \cdot \mathrm{min}^{-1}$. Foi utilizada uma taxa de resfriamento de $5^{\circ} \mathrm{C} \cdot \mathrm{min}^{-1} \mathrm{em}$ todos os casos.

\section{Imobilização dos catalisadores na espuma cerâmica}

Os catalisadores $\mathrm{ZnAl}_{2} \mathrm{O}_{4}[14], \mathrm{ZnFe}_{2} \mathrm{O}_{4}[19]$ e $\mathrm{Zn}_{2} \mathrm{SnO}_{4}$ [20] foram preparados pela rota hidrotérmica e a metodologia de síntese e as respectivas caracterizações encontram-se apresentadas em trabalhos prévios. Para a impregnação dos catalisadores $\mathrm{ZnAl}_{2} \mathrm{O}_{4}, \mathrm{ZnFe}_{2} \mathrm{O}_{4}$ e $\mathrm{Zn}_{2} \mathrm{SnO}_{4}$ na espuma cerâmica, utilizado $5 \mathrm{~g}$ de catalisador foi adicionado em 50 $\mathrm{mL}$ de água destilada, sob vigorosa agitação magnética. Essa foi realizada através do método de dip coating, sendo utilizado o protótipo feito no Laboratório de Propriedades Físicas de Matérias Cerâmicos (LaPFiMC) da Universidade Federal do Rio Grande do Norte. O equipamento foi programado com uma velocidade de descida/subida de $10 \mathrm{~mm} \cdot \mathrm{min}^{-1} \mathrm{e}$ um tempo de permanência submerso na suspensão de $1 \mathrm{~min}$. Após as impregnações, as espumas cerâmicas passaram por um tratamento térmico de $600{ }^{\circ} \mathrm{C}$ em atmosfera oxidante (ar), para auxiliar a fixação dos catalisadores na superfície da espuma cerâmica. $\mathrm{O}$ tratamento térmico foi realizado em um forno mufla a uma taxa de aquecimento de $5{ }^{\circ} \mathrm{C} \cdot \mathrm{min}^{-1}$, mantendo por $1 \mathrm{~h}$ após atingir a temperatura desejada.

\section{Análises de caracterização}

O comportamento reológico da suspensão cerâmica foi medido usando um reômetro Haake Polylab OS System da ThermoHaake a uma taxa de cisalhamento de 0 a $500 \mathrm{~s}^{-1}$ a $30^{\circ} \mathrm{C}$. Resistência à flexão em quatro pontos de cada amostra de espuma produzida foi medida em uma máquina de ensaios mecânicos de bancada (Zwick/Roell BZ 2.5/TS1T 1), com uma velocidade de carregamento de $0,5 \mathrm{~mm} \cdot \mathrm{min}^{-1}$.

A morfologia dos materiais preparados foi analisada em um microscópio eletrônico de varredura SSX550 Superscan (Shimadzu), no qual se observou a estrutura dos filamentos e os defeitos existentes.

A fase da espuma cerâmica e dos catalisadores impregnados foi avaliada por difração de raios $\mathrm{X}$ em um difratômetro XRD 6000 (Shimadzu), e com o complemento de uma análise semiquantitativa de espectroscopia de energia dispersiva (EDS).

\section{RESULTADOS E DISCUSSÃO}

Uma das preocupações na utilização do método da preparação das espumas está na avaliação da suspensão 


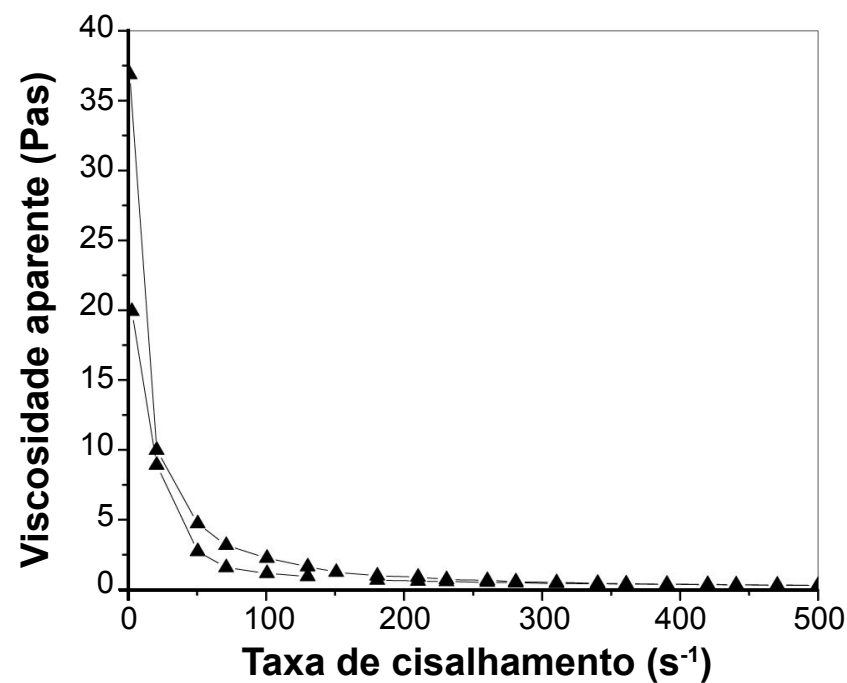

Figura 1: Viscosidade aparente versus taxa de cisalhamento. [Figure 1: Apparent viscosity versus shear rate.]

de partida. Esta deve ter características reológicas apropriadas, ou seja, deve ser suficientemente fluida para ser parcialmente removida quando passada pelos rolos, mas também suficientemente viscosa após o revestimento a ponto de evitar o gotejamento permitindo um recobrimento uniforme dos filamentos da espuma de poliuretano [21]. Por conseguinte suspensões cerâmicas que exibam comportamento pseudoplástico são necessárias para revestir o molde polimérico com eficiência [22].

Através das análises de comportamento reológico foi possível observar a presença do comportamento pseudoplástico na suspensão cerâmica utilizada. A Fig.1 mostra o comportamento reológico da suspensão através da viscosidade em função da taxa de cisalhamento aplicada. A curva mostra uma diminuição da viscosidade aparente com o aumento da taxa de cisalhamento, evidenciando um comportamento típico pseudoplástico, o qual é necessário para a presente proposta [23]. Esse comportamento é conseguido através do uso de ligantes em combinação com

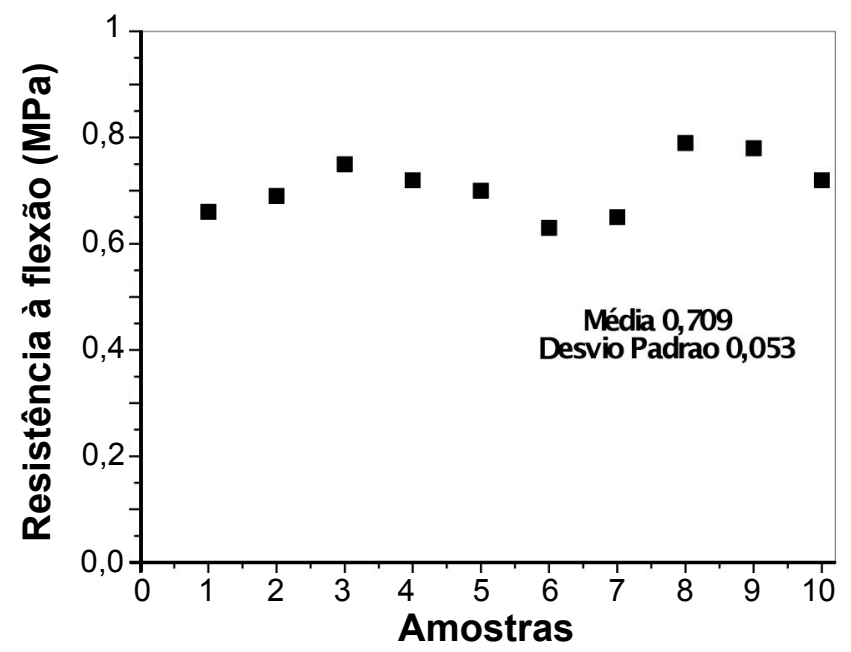

Figura 2: Resistência mecânica da espuma cerâmica. [Figure 2: Mechanical strength of the ceramic foam.] agentes dispersantes [21].

A Fig. 2 representa o gráfico da resistência mecânica à flexão em quatro pontos da espuma cerâmica. Para resultados mais representativos, foram testadas 10 amostras. Os valores variaram entre 0,6 e $0,8 \mathrm{MPa}$, resultando num baixo desvio padrão de 0,053 , o que demonstra a boa reprodutibilidade do método de preparação das espumas cerâmicas usado neste trabalho.

A literatura reporta que a resistência mecânica depende tanto do tamanho das células que compõem a estrutura da cerâmica reticulada, como também do grau de interconectividade entre elas e dos defeitos existentes na estrutura [21]. Esses valores de resistência estão associados à quantidade de defeitos nos filamentos que formam estrutura celular da espuma. As micrografias da espuma cerâmica preparada neste trabalho estão apresentadas na Fig. 3. Observa-se um material com os poros abertos e com filamentos finos e interligados, muito próximo da estrutura original da esponja de poliuretano. Pode-se observar
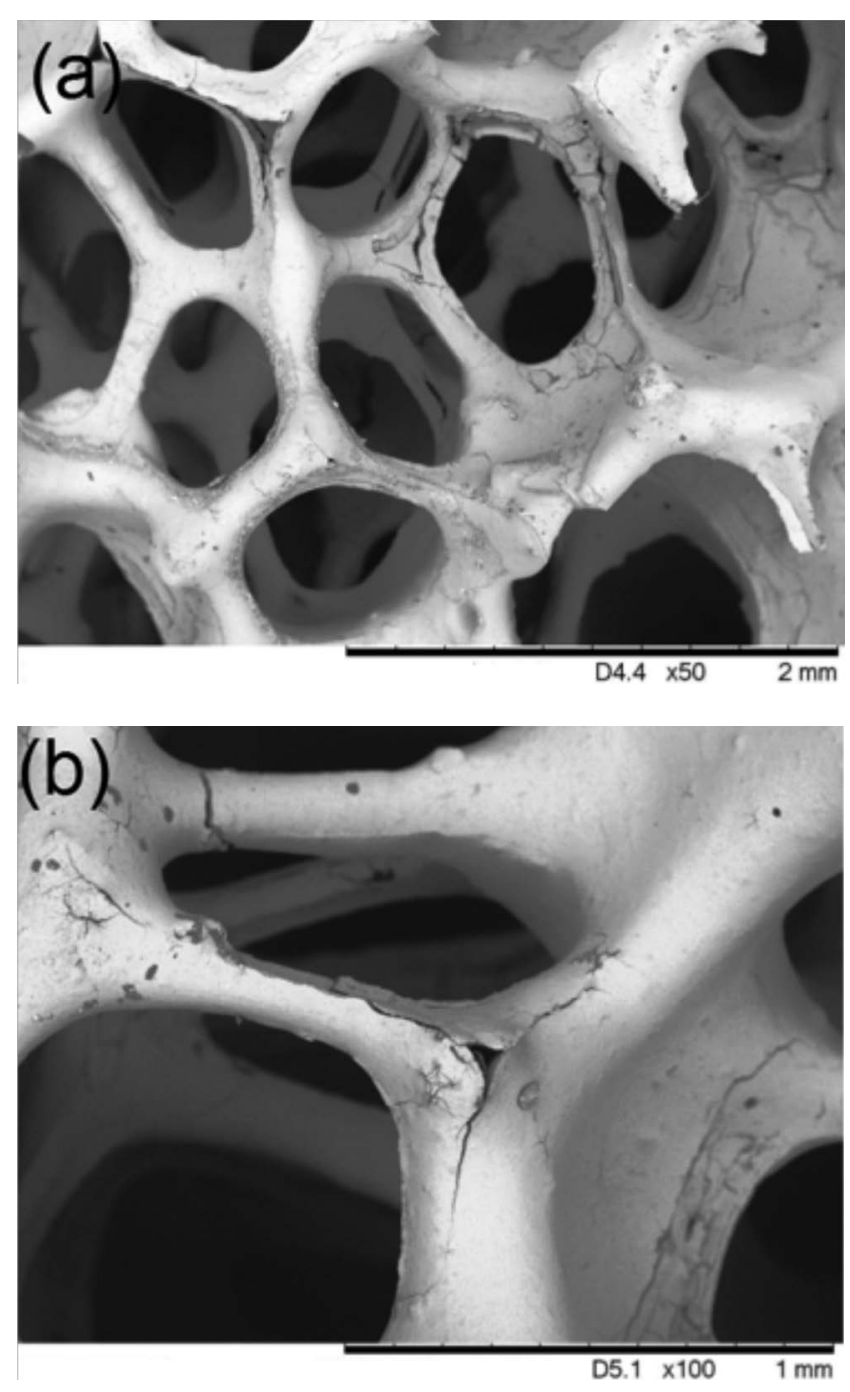

Figura 3: Micrografias obtidas em microscópio eletrônico de varredura da espuma cerâmica. (a) ampliação de 50x e (b) 100x. [Figure 3: SEMmicrographs of the ceramicfoam. (a) magnification: 50x and (b)100x.] 


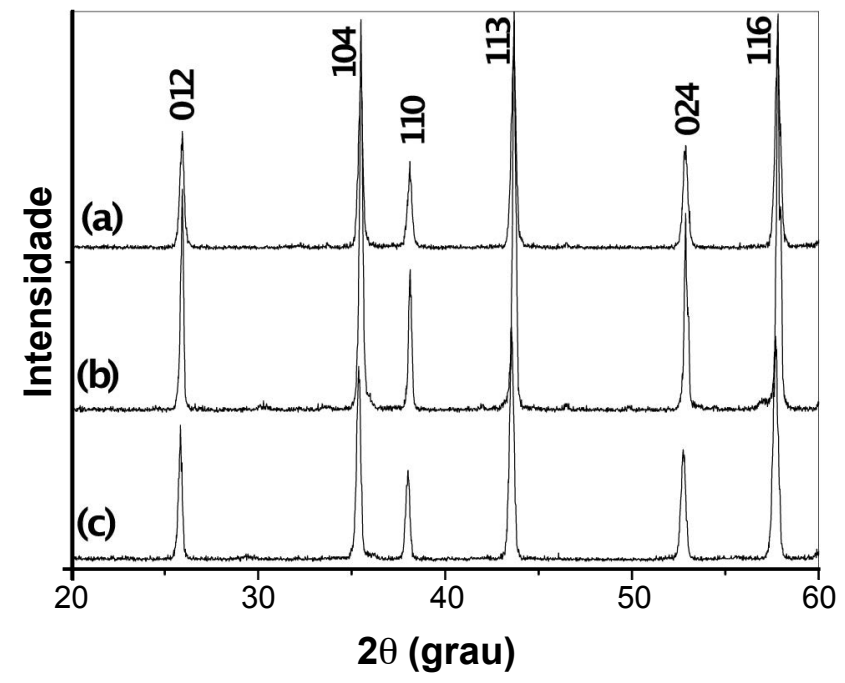

Figura 4: Difratogramas de raios $\mathrm{X}$ da espuma cerâmica impregnada com diferentes catalisadores: (a) $\mathrm{ZnAl}_{2} \mathrm{O}_{4} / \mathrm{Al}_{2} \mathrm{O}_{3}$, (b) $\mathrm{ZnFe}_{2} \mathrm{O}_{4} /$ $\mathrm{Al}_{2} \mathrm{O}_{3} \mathrm{e}(\mathrm{c}) \mathrm{Zn}_{2} \mathrm{SnO}_{4} / \mathrm{Al}_{2} \mathrm{O}_{3}$.

[Figure 4: X-ray diffraction patterns of ceramic foam impregnated with different catalysts: (a) $\mathrm{ZnAl}_{2} \mathrm{O}_{4} / \mathrm{Al}_{2} \mathrm{O}_{3}$, (b) $\mathrm{ZnFe}_{2} \mathrm{O}_{4} / \mathrm{Al}_{2} \mathrm{O}_{3}$ and (c) $\mathrm{Zn}_{2} \mathrm{SnO}_{4} / \mathrm{Al}_{2} \mathrm{O}_{3}$.]
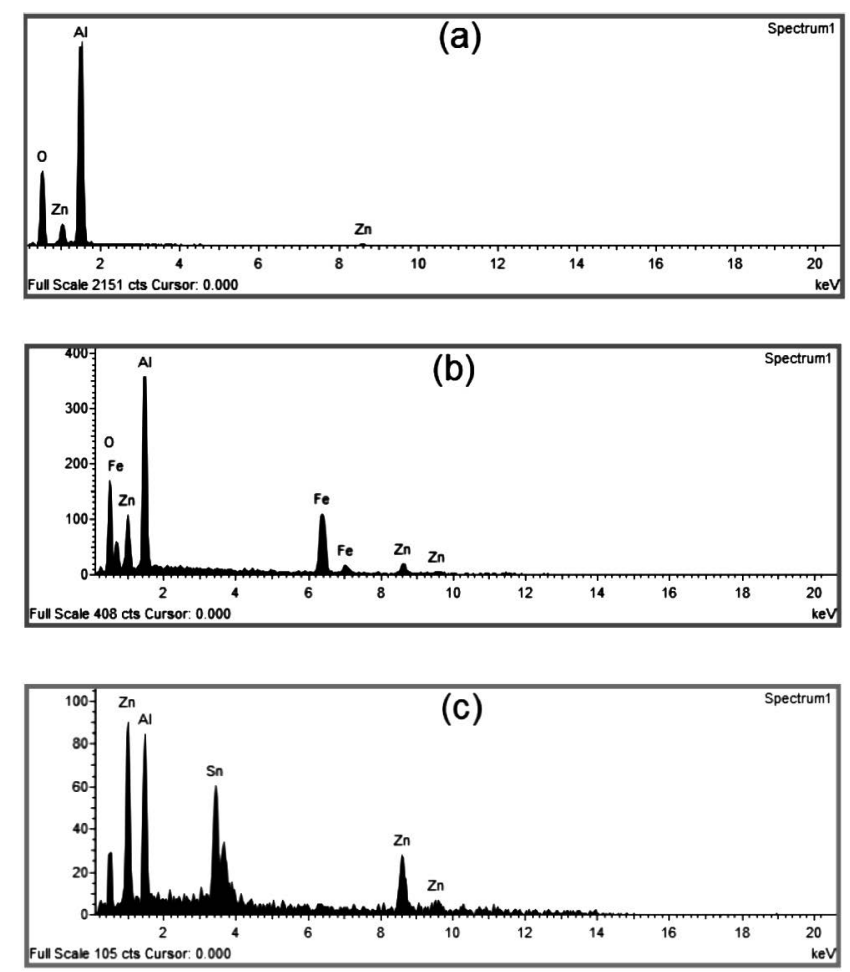

Figura 5: EDS das espumas cerâmicas impregnadas com (a) $\mathrm{ZnAl}_{2} \mathrm{O}_{4}$, (b) $\mathrm{ZnFe}_{2} \mathrm{O}_{4}$ e (c) $\mathrm{Zn}_{2} \mathrm{SnO}_{4}$.

[Figure 5:EDS of the ceramic foams impregnated with a) $\mathrm{ZnAl}_{2} \mathrm{O}_{4}$, (b) $\mathrm{ZnFe}_{2} \mathrm{O}_{4}$ and (c) $\mathrm{Zn}_{2} \mathrm{SnO}_{4}$.]

também, algumas falhas, tais como fendas laterais e no interior dos filamentos. Estes defeitos são provenientes da etapa de queima no processamento do material, que ocorre pelo acúmulo de pressão no interior dos filamentos durante a volatilização do molde polimérico pelo tratamento térmico [21]. A presença destes defeitos contribuiu para a
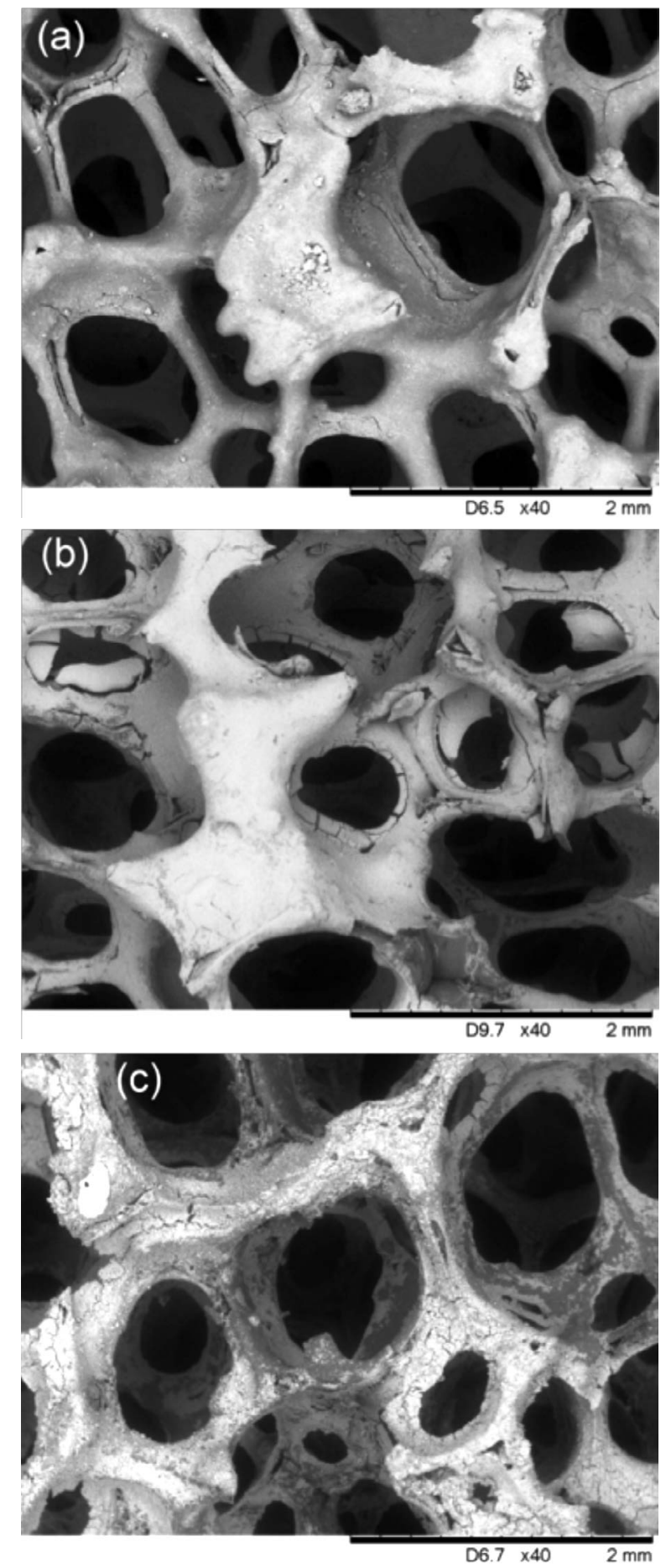

Figura 6: Micrografias obtidas em microscópio eletrônico de varredura das espumas cerâmicas impregnada com (a) $\mathrm{ZnAl}_{2} \mathrm{O}_{4}$, (b) $\mathrm{ZnFe}_{2} \mathrm{O}_{4}$ e (c) $\mathrm{Zn}_{2} \mathrm{SnO}_{4}$. (Ampliação de 40x).

[Figure 6: SEM micrographs of ceramic foams impregnated with (a) $\mathrm{ZnAl}_{2} \mathrm{O}_{4}$, (b) $\mathrm{ZnFe}_{2} \mathrm{O}_{4}$ and (c) $\left.\mathrm{Zn}_{2} \mathrm{SnO}_{4} \cdot\right]$

diminuição da resistência mecânica destes materiais [24]. Por outro lado, esses defeitos contribuem para o aumento da área superficial dessa espuma cerâmica, propriedade essa de suma importância na área da catálise.

Na Fig. 4 estão apresentados os difratogramas de raios $\mathrm{X}$ das três amostras de suporte cerâmico impregnadas com 
os catalisadores. Todos os picos dos difratogramas estão indexados a uma alumina de estrutura cúbica, o qual está em boa concordância com o arquivo JCPDS 81-1667. Os picos característicos a $2 \Theta$ de $25,57^{\circ}, 35,14^{\circ}, 37,58^{\circ}$, $43,35^{\circ}, 52,53^{\circ}$ e $57,50^{\circ}$ são correspondentes ao planos de difração da alumina a (012), (104), (110), (113), (024) e (116), respectivamente. Isso indica que a fase relativa aos catalisadores não apareceu nos difratogramas, e isso está atribuído à baixa concentração desses materiais impregnados no suporte cerâmico, pois foi realizado apenas um fino recobrimento na superfície da espuma, não sendo assim, possível identificá-los por essa análise. Dessa forma, com o intuito de verificar se os catalisadores foram impregnados na superfície da espuma cerâmica, foi realizada uma análise de EDS.

Pela análise de EDS, Fig. 5, foi possível observar os elementos que formam os catalisadores impregnados sobre a superfície das espumas cerâmicas. Na Fig. 5a aparecem o metais $\mathrm{Zn}$ e $\mathrm{Al}$, relativos à amostra $\mathrm{ZnAl}_{2} \mathrm{O}_{4}$, enquanto que na Fig. 5b os metais $\mathrm{Zn}$ e $\mathrm{Fe}$, relativos à amostra $\mathrm{Zn}_{2} \mathrm{FeO}_{4}$, e por último (Fig. 5c) os metais $\mathrm{Zn}$ e $\mathrm{Sn}$, que constituem a amostra $\mathrm{Zn}_{2} \mathrm{SnO}_{4}$. Cabe ressaltar que o $\mathrm{Al}$ aparece em todas as análises, pois corresponde ao alumínio que forma o porta-amostra. Assim, na amostra $\mathrm{ZnAl}_{2} \mathrm{O}_{4}$ (Fig. 5a), o Al constituinte do catalisador está sobreposto ao $\mathrm{Al}$ do portaamostra.

A Fig. 6 apresenta as micrografias do suporte cerâmico após as impregnações com os catalisadores. É possível observar a distribuição dos catalisadores nos filamentos das espumas cerâmicas após as impregnações. A espuma impregnada com $\mathrm{ZnAl}_{2} \mathrm{O}_{4}$ (Fig. 6a) apresenta um fino recobrimento da superfície da espuma com poucos aglomerados entre os filamentos e pouca distribuição do catalisador na superfície do material. Já para o material da Fig. 6b é possível observar a distribuição do catalisador $\mathrm{ZnFe}_{2} \mathrm{O}_{4}$ na superfície da espuma, verificando um recobrimento uniforme do catalisador na superfície do material, como também um excesso do material entre os filamentos. Já para o material da Fig. 6c é possível observar um fino recobrimento esbranquiçado, relativo ao catalisador imobilizado sobre a superfície do suporte cerâmico.

\section{CONCLUSÕES}

Foram obtidas esponjas de alumina a partir de esponjas de poliuretano mediante a utilização do método da réplica, no qual foi possível obter estruturas cerâmicas com características próximas ao molde polimérico. A suspensão utilizada apresentou comportamentos característicos pseudoplásticos. Na caracterização das esponjas cerâmicas observou-se que o material obtido apresentou uma adequada resistência mecânica, sendo essa característica importante para a utilização do material como suporte de catalisadores. Através da análise de EDS foi possível confirmar a impregnação dos catalisadores $\mathrm{ZnAl}_{2} \mathrm{O}_{4}, \mathrm{ZnFe}_{2} \mathrm{O}_{4}$ e $\mathrm{Zn}_{2} \mathrm{SnO}_{4}$ na superfície da esponja cerâmica, evidenciando que a técnica de dip coating pode ser utilizada para a imobilização desses catalisadores em superfícies porosas cerâmicas.

\section{AGRADECIMENTOS}

Os autores agradecem o apoio da CAPES, via Edital PROCAD-238/2007, Proc. 23038.022051/2008-26.

\section{REFERÊNCIAS}

[1] G. Plesch, M. Gorbár,U. F. Vogt, K. Jesenák, M.Vargová, Mater. Lett. 63 (2009) 461.

[2] A. M. Ramirez, K. D. Belie, T. Mantyla, E. Levanen, Build. Environ. 45 (2010) 832.

[3] F. S. Ortega, K. M. Rocha, M. Zaiat, V. C. Pandolfelli, Cerâmica 47 (2001) 199.

[4] S.-A. Seyed-Reihani, G. S. Jackson, Appl. Catal. A: Gen. 353 (2009) 181.

[5] T. Granato, F. Testa, R. Olivo, Microp. Mesop. Mater. 153 (2012) 236.

[6] M. I. Domínguez, M. Sánchez, M. A. Centeno, M. Montes, J. A. Odriozola, Appl. Catal. A: Gen. 302 (2006) 96. [7] Y. Han, H. Kim, M. Tong, Mater. Chem. Phys. 134 (2012) 26.

[8] M. Scheffler, P. Colombo, "Cellular ceramics: structure, manufacturing, properties and applications", Weinheim: Wiley-VCH (2005).

[9] R. C. O. Romano, V. C. Pandolfelli, Cerâmica 52 (2006) 213.

[10] J. A. Toledo-Antonio, N. Nava, M. Martínez, X. Bokhimi, Appl. Catal. A: Gen. 234 (2002) 137.

[11] M. Vijayaraj, C. S. Gopinath, J. Catal. 241 (2006) 83.

[12] M. Lenarda, M. Casagrande, E. Moretti, L. Storaro, R. Frattini, S. Polizzi, Catal. Lett. 114 (2007) 79.

[13] G. Fan, Z. Gu, L. Yang, F. Li, Chem. Eng. J. 155 (2009)534

[14] E. L. Foletto, S. Battiston, J. M. Simões, M. M. Bassaco, L. S. F. Pereira, E. M. M. Flores, E. I. Müller, Micropor. Mesopor. Mater. 163 (2012)29.

[15] E. L. Foletto, J. M. Simões, M. A. Mazutti, S. L. Jahn, E. I. Müller, Pereira, L. S. F. Pereira, E. M. M. Flores, Ceram. Int. 39 (2013) 4569.

[16] J. Livage, Mater. Sci. Forum 152-153 (1994) 43.

[17] J. S. Woyansky, C. E. Scott, W. P. Minnear, Am. Ceram. Soc. Bull. 71 (1992) 1674.

[18] H. Haugen, J. Will, A. Kohler, U. Hopfner, J. Aigner, E. Wintermantel, J. Eur.Ceram. Soc. 24 (204) 661.

[19] C. G. Anchieta, F. M. Stringhini, D. Sallet, E. L. Foletto, S. S. da Silva, O. Chiavone-Filho, C. A. O. do Nascimento, Ceram. Int.,DOI: 10.1016/j.ceramint.2013.08.074.

[20] E. L. Foletto, S. L. Jahn, R. F. P. M. Moreira, J. Appl. Electrochem. 40 (2010) 59.

[21] A. R. Studart, U. T. Gonzenbach, E. Tervoort, L. J. Gauckler, J. Am. Ceram. Soc. 89 (2006) 1771.

[22] D. R. Dinger, "Rheology for ceramists", Dinger Ceramic Cons. Serv., Morris Publ. (2002).

[23] C. M. Gomes, Avaliação do comportamento reológico de suspensões cerâmicas triaxiais utilizando abordagem do 
delineamento de misturas, 127 f., Tese Dr. (Pós-graduação em Ciência e Engenharia de Materiais), Universidade Federal de Santa Catarina, Florianópolis, SC, Brasil (2004).
[24] X. Yao, S. Tan, Z. Huang, D. Jiang, Ceram. Int. 32 (2006) 137.

(Rec. 24/05/2013, Rev. 16/10/2013, Ac. 17/10/2013) 